\title{
Effectiveness of post-operative management strategies for adults with dementia following hip fracture surgery (Protocol)
}

Smith TO, Hameed YA, Henderson C, Cross JL, Sahota O, Fox C

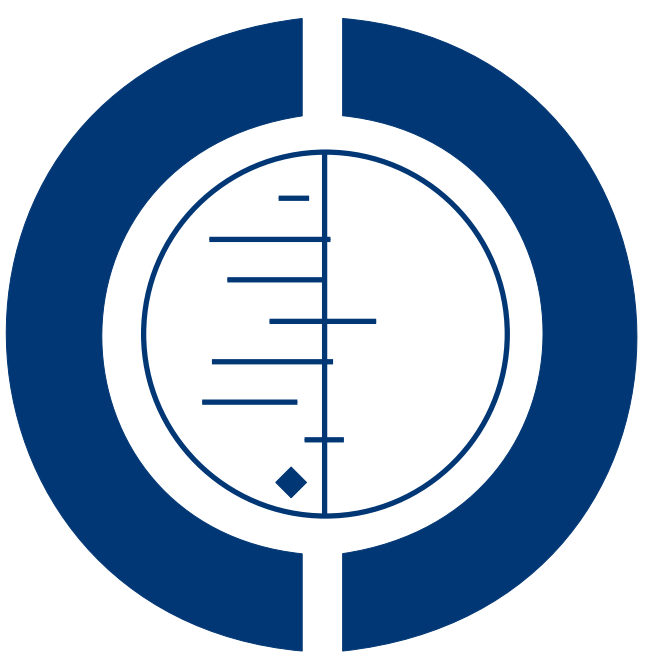

THE COCHRANE

COLLABORATION $^{\circledR}$

This is a reprint of a Cochrane protocol, prepared and maintained by The Cochrane Collaboration and published in The Cochrane Library 2013, Issue 6

http://www.thecochranelibrary.com

\section{WILEY}

Effectiveness of post-operative management strategies for adults with dementia following hip fracture surgery (Protocol) Copyright $\odot 2013$ The Cochrane Collaboration. Published by John Wiley \& Sons, Ltd. 
TABLE OF CONTENTS

HEADER . . . . . . . . . . . . . . . . . . . . . . . . . . . . . . . . . . . . 1

ABSTRACT . . . . . . . . . . . . . . . . . . . . . . . . . . . . . . . . . . . . . . . 1

BACKGROUND . . . . . . . . . . . . . . . . . . . . . . . . . . . . . . . . . . . .

OBJECTIVES . . . . . . . . . . . . . . . . . . . . . . . . . . . . . . . . . . . . . . .

METHODS . . . . . . . . . . . . . . . . . . . . . . . . . . . . . . . . . . . . . . . .

ACKNOWLEDGEMENTS . . . . . . . . . . . . . . . . . . . . . . . . . . . . . . . . . . . . . . . .

REFERENCES . . . . . . . . . . . . . . . . . . . . . . . . . . . . . . . . . . . . . . 6

APPENDICES . . . . . . . . . . . . . . . . . . . . . . . . . . . . . . . . . . . . . 9

CONTRIBUTIONS OF AUTHORS . . . . . . . . . . . . . . . . . . . . . . . . . . . . . . . . . . . . . . . . . . . .

DECLARATIONS OF INTEREST . . . . . . . . . . . . . . . . . . . . . . . . . . . . . . . . . 10

SOURCES OF SUPPORT . . . . . . . . . . . . . . . . . . . . . . . . . . . . . . . . . . . . . . . . .

Effectiveness of post-operative management strategies for adults with dementia following hip fracture surgery (Protocol)

Copyright $\odot 2013$ The Cochrane Collaboration. Published by John Wiley \& Sons, Ltd. 


\title{
[Intervention Protocol] \\ Effectiveness of post-operative management strategies for adults with dementia following hip fracture surgery
}

\author{
Toby O Smith ${ }^{1}$, Yasir A Hameed ${ }^{2}$, Catherine Henderson ${ }^{3}$, Jane L Cross ${ }^{4}$, Opinder Sahota ${ }^{5}$, Chris Fox ${ }^{6}$ \\ ${ }^{1}$ Faculty of Medicine and Health Sciences, University of East Anglia, Norwich, UK. ${ }^{2}$ Psychiatry, Norfolk and Suffolk NHS Foundation \\ Trust, Hellesden Hospital, Norwich, UK. ${ }^{3}$ Personal Social Services Research Unit, London School of Economics and Political Science, \\ London, UK. ${ }^{4}$ School of Allied Health Professions, University of East Anglia, Norwich, UK. ${ }^{5}$ Healthcare of Older People, Nottingham \\ University Hospitals NHS Trust, QMC, Nottingham, UK. ${ }^{6}$ Norwich Medical School, Norwich, UK
}

Contact address: Toby O Smith, Faculty of Medicine and Health Sciences, University of East Anglia, Queen's Building, Norwich, Norfolk, NR4 7TJ, UK. toby.smith@uea.ac.uk.

Editorial group: Cochrane Dementia and Cognitive Improvement Group.

Publication status and date: New, published in Issue 6, 2013.

Citation: Smith TO, Hameed YA, Henderson C, Cross JL, Sahota O, Fox C. Effectiveness of post-operative management strategies for adults with dementia following hip fracture surgery. Cochrane Database of Systematic Reviews 2013, Issue 6. Art. No.: CD010569. DOI: 10.1002/14651858.CD010569.

Copyright (C) 2013 The Cochrane Collaboration. Published by John Wiley \& Sons, Ltd.

\begin{abstract}
A B S T R A C T
This is the protocol for a review and there is no abstract. The objectives are as follows:

To assess the effects of different post-operative management strategies for people with dementia following hip fracture surgery, with a bias towards dementia and cognitive or behavioural outcomes.
\end{abstract}

\section{B A C K G R O U N D}

\section{Description of the condition}

The hip joint is the articulation between the thigh bone (femur) and the pelvis. The term 'hip fracture' encompasses all fractures of the upper (proximal) part of the thigh bone (femur). Hip fractures are commonly divided into two types: intracapsular fractures, which represent those that occur within or proximal to the attachment of the hip joint capsule to the femur; and extracapsular, which represent fractures occurring outside or lower (distal) to the hip joint capsule (Parker 2010). Hip fracture is a common injury in the elderly population.

The majority of people undergo hip surgery following hip fracture (Uzoigwe 2012). The location of the fracture, stability and degree of comminution (number of pieces the bone breaks into) determine which operative procedure should be undertaken in order to repair the hip fracture. The aim of surgery, irrespective of the type of operation, is to reduce pain, facilitate early weight-bearing mobility to improve outcomes, and to facilitate independence in activities of daily living, such as bathing, dressing, and continence (Handoll 2009). A delay in surgical intervention is known to be a key factor in producing poorer outcomes (Vidal 2012).

The annual hip fracture incidence rate has been estimated as 1.29/ 1000 person-years in males and 2.24/1000 person-years in females (Adams 2012). This figure is likely to rise over the next few years as the population is increasing in age (Cummings 2002). It is the most common physical rehabilitation condition for older adults (Lenze 2007), seen in both those who are cognitively intact and those with all degrees of cognitive impairment, and is associated with

Effectiveness of post-operative management strategies for adults with dementia following hip fracture surgery (Protocol)

Copyright $\odot 2013$ The Cochrane Collaboration. Published by John Wiley \& Sons, Ltd. 
significant pain and loss of independence and function (Morrison 2000). Thirty-three per cent to $37 \%$ of patients return to their prior level of function by six months, including those needing assistance, but only $24 \%$ are independent in locomotion at by six months (Magaziner 2002).

Dementia is a global loss of cognitive and intellectual functioning, which gradually interferes with social and occupational performance (Lieberman 2006; McGilton 2012). It is a common condition with a significant impact on society. Hip fracture is nearly three times more common in people with dementia than in people without dementia (Zhao 2012). It is expected that the incidence of patients with dementia and hip fracture will increase during the next 25 years (Adunsky 2003; Knapp 2007). Health and social care expenditure in England on people with dementia in the year following admission for fractured neck of femur has been estimated to be in excess of GBP 1 billion (GBP 1037 million in 2005 to 2006 prices), about GBP 0.4 billion higher than expenditure on those without dementia (Henderson 2007). This was estimated as equating to approximately GBP 34,200 per person per annum for those without dementia and GBP 40,300 per person per annum for people with dementia (Henderson 2007).

\section{Description of the intervention}

The provision of high-quality care for people following hip fracture has been identified as a major clinical need in the United Kingdom and elsewhere. This has been exemplified in the United Kingdom through the development of national guidelines (NICE 2011), the introduction of specific financial incentives for highquality care through the 'Best Practice Tariff' (NICE 2011), and the national audit of standards of care provision to this population through the National Hip Fracture Database (National Hip Fracture Database 2013). For all hip fracture patients, initial management is usually provided in an acute hospital setting, where the person undergoes an operation for their hip fracture. Best practice often includes shared orthopaedic and geriatric (sometimes termed ortho-geriatric) care pre- and post-operatively to ensure that patients are medically fit for surgery and to monitor and manage any post-operative medical complications that may develop (Dy 2012). These may include pneumonia, anaemia, dehydration, pressure sores, or cardiovascular complications (Dy 2012; Jameson 2012). During the initial hip fracture admission, or index admission (Drummond 2005), health professionals such as nurses, pharmacists, occupational therapists, physiotherapists, social workers, and dieticians may be involved in the patient's recovery and rehabilitation (Kammerlander 2010; Stenvall 2012). Depending on their home circumstances and their post-operative functional capabilities, patients may be discharged directly to the residential setting they lived in, with or without community or out-patient rehabilitation, or may be transferred to an in-patient rehabilitation unit to receive continued multi-professional rehabilitation. Patients will remain in this rehabilitation setting until they are sufficiently independent to be discharged to their preadmission residence or, if this is not achievable, they may be provided with residential or nursing home care (Hashmi 2004).

Over the past 15 years, developments in the management of people with hip fracture have been advanced (Cameron 2000). This has particularly been seen for those with dementia, who have specific and complex care needs (Cameron 2000; Dy 2012). Over this period, research reports and subsequent clinical guidelines have recommended a number of interventions to improve outcomes for this group of patients (NICE 2011). These have included specific medical management by an ortho-geriatrician on specified hip-fracture wards, which is considered to enhance interdisciplinary team working; improvement of communication between health and social agencies (Kammerlander 2010; Stenvall 2012); provision of dedicated functional rehabilitation interventions across acute hospital and community rehabilitation settings (Al-Ani 2010; Huusko 2000); monitoring of post-operative complications including pressure sores (Söderqvist 2007); and optimisation of nutritional levels for this group of patients (Hershkovitz 2010). Specific rehabilitation strategies for this population have included enhanced rehabilitation with respect to orientation to the environment, clues, reminiscence and structured, familiarised routines. Such interventions can be delivered in a variety of healthcare and domiciliary settings.

\section{How the intervention might work}

The interventions that have been proposed to improve the management of people with dementia who have suffered a hip fracture have been advocated to improve communication between healthcare professionals and provide generic and wider healthcare expertise than may conventionally be found on an orthopaedic ward or in a rehabilitation setting (Söderqvist 2007). Recommended interventions have also included specifically targeting interventions and resources for this population, who have greater and more complex healthcare needs (Söderqvist 2007). These factors are acknowledged as possible explanations why a specific, targeted management programme for people with dementia following hip fracture may be advantageous over conventional, non-specific postoperative management (Handoll 2009).

\section{Why it is important to do this review}

More than three quarters of a million people in the UK have dementia. One in four National Health Service (NHS) beds are occupied by someone with dementia. Fractured hips and falls are the commonest reasons for hospital admission. People with dementia who sustain a hip fracture have more complex health problems with complications, disabilities, and social needs. Whilst previous reviews have examined the rehabilitation of people following hip fracture, none have specifically assessed the specialist rehabilitation 
strategies for those who have dementia. Since this population has complex care needs, and makes a major demands on healthcare services, this focused review of the literature is warranted.

In this population, factors such as depression, motivation, pain, and cognitive impairment have been cited as impacting on clinical outcomes (Lenze 2007). Pain has been acknowledged as a particular problem since if pain management is inadequate, due to poor assessment, negative post-operative outcomes and complications such as pneumonia, atrophy, and thromboembolism can occur (Egbert 1996; Feldt 1998; Morrison 1998). These factors may adversely impact on the ability of a person to return to functional independence, the discharge destination, the length of their in-patient hospital stay and rehabilitation requirements. The resulting negative consequences, therefore, have a health economic impact, at a personal and a systems level. People who sustain a hip fracture and have dementia experience longer hospitalisations with poorer outcomes, such as higher mortality and morbidity rates, and have a greater risk of requiring nursing home placement and poorer functional recovery (Gruber-Baldini 2003; Magaziner 1990; Steiner 1997). However, whilst various interventions have been supported for the targeted rehabilitation of people with dementia who experience a hip fracture (Al-Ani 2010; Huusko 2000), these are more expensive than conventional post-operative management following hip fracture (Lenze 2007). More evidence is needed on the relationship between the processes and outcomes of post-operative care, length of stay, and costs in the general population of hip fracture patients (Hunt 2009), and in particular in the subpopulation of people with dementia (Henderson 2007). Decisions as to whether to allocate limited health and social care resources to these new interventions can be informed by economic evaluation, the comparative analysis of outcomes and the costs of alternative treatment programmes (Drummond 2005).

Previous reviews have examined the literature on the use of management strategies for people with dementia who experience a hip fracture (Allen 2012; Handoll 2009). These have focused on clinical and functional outcomes. No reviews have specifically assessed the impact of such management programmes on behavioural, cognitive, or dementia-related outcomes for this population, nor on the relationship between these outcomes and resource use and costs. The purpose of this review is therefore to answer these important questions.

\section{O B J E C T IVES}

To assess the effects of different post-operative management strategies for people with dementia following hip fracture surgery, with a bias towards dementia and cognitive or behavioural outcomes.

\section{Criteria for considering studies for this review}

\section{Types of studies}

We will include randomised, quasi-randomised (method of allocating participants to a treatment which is not strictly random, for example by hospital number) or cluster-randomised controlled clinical trials published in any language, evaluating the effectiveness of different post-operative management strategies for people with dementia following hip fracture surgery. We will include studies of costs and cost-effectiveness accompanying eligible effectiveness studies of post-operative management strategies for people with dementia following hip fracture surgery (Shemilt 2008).

\section{Types of participants}

We will include all people if they are aged 65 years or over, have been diagnosed with any form of dementia, and have undergone hip fracture surgery for a proximal femoral fracture. We will exclude studies where over $30 \%$ of participants presented with a mid-shaft or distal femoral fracture. Dementia should have been diagnosed using a validated instrument such as the Diagnostic and Statistical Manual IV (American Psychiatric Association 1994) or International Classification of Diseases 10th revision (ICD-10) (World Health Organization 2007). We will contact corresponding authors for further information if the method of diagnosing dementia is not stipulated in the original study. Participants may be resident in the community, in care homes, or hospitals for shortor long-term care.

\section{Types of interventions}

We will include any form of post-operative management or rehabilitation programme following a hip fracture that is intended specifically for people with dementia or cognitive impairment. This may include post-operative recovery on a specialist orthogeriatric ward and enhanced rehabilitation with respect to: orientation to the environment, clues, reminiscence and structured, familiarised routines undertaken. Interventions may be delivered in acute hospital environments, community health or rehabilitation centres, in community centres or non-health settings, or domiciliary in people's homes and residences.

For comparison, we will compare study interventions to routine post-operative and rehabilitation management. Neither the intervention nor the control will be known until the search is conducted.

\section{Types of outcome measures}


- Cognitive function as assessed using (for example): Alzheimer's Disease Assessment Scale Cognitive Subscale (ADASCOG) (Rosen 1984), Mini-Mental State Examination (MMSE) (Folstein 1975), Abbreviated Mental Test (Hodkinson 1972), Addenbrooke's Cognitive Examination Revised (ACE-R) (Mathuranath 2005), Montreal Cognitive Assessment (MoCA) (Nasreddine 2005), Hopkins Verbal Learning Test (HVLT-R) (Brandt 1991), the Informant Questionnaire on Cognitive Decline in the Elderly (IQCODE) (Jorm 1989).

\section{Secondary outcomes}

- Cognitively determined function as assessed with tools such as the: Barthel Index (Mahoney 1965), Nottingham Extended Activities of Daily Living Scale (Nouri 1987), Oxford Hip Score (Dawson 1996), and the Bristol Activities of Daily Living Score (Bucks 1996).

- Behaviour as assessed using: Neuropsychiatric Inventory (NPI) (Cummings 1994), Cohen-Mansfield Agitation Inventory (CMAI) (Cohen-Mansfield 1986).

- Quality of Life as assessed using: the Short Form-36 (Ware 1992), Bath Assessment of Subjective Quality of Life in Dementia (BASQID) (Trigg 2007), DEMQOL (Smith 2005), Short Form-12 (Ware 1996), EuroQol (EQ)-5D (EuroQol Group 1990), and Health Utility Index (Feeny 2002) instruments.

- Tools assessing pain, from any cause, using methods suited to patients with dementia, such as the Pain Assessment in Advanced Dementia (PAINAD) (Warden 2003).

- Mortality.

- Complications such as deep vein thrombosis, pressure sores, pneumonia.

- Use of health and social care resources: hospital length of stay, hospital re-admissions, discharge destination (to pre-injury setting, residential or nursing home care), use of primary and community care support services including general physician (GP) visits, medications and tests prescribed, also community and residential rehabilitation.

- Costs of hospitalisation, hospital re-admission, health and social care support in the community or in residential or nursing home care, and costs to people with dementia who have had a hip fracture and to their carers (such as travel, carers' lost production).

\section{Search methods for identification of studies}

We will perform the search methods in accordance with the latest version in the Cochrane Handbook for Systematic Reviews of Interventions (Lefebvre 2011).

\section{Electronic searches}

We will search ALOIS (www.medicine.ox.ac.uk/alois), the Cochrane Dementia and Cognitive Improvement Group Specialized Register.

ALOIS is maintained by the Trials Search Co-ordinator and contains dementia and cognitive improvement studies identified from the following.

1. Monthly searches of a number of major healthcare databases: MEDLINE, EMBASE, CINAHL, PsycINFO, and LILACS.

2. Monthly searches of a number of trial registers: metaRegister of Controlled Trials; Umin Japan Trial Register; WHO Clinical Trials Registry Platform portal (which covers ClinicalTrials.gov; ISRCTN; Chinese Clinical Trial Register; German Clinical Trials Register; Iranian Registry of Clinical Trials; the Netherlands National Trials Register, plus others).

3. Quarterly search of the Central Register of Controlled Trials (CENTRAL) in The Cochrane Library.

4. Monthly searches of a number of grey literature sources: ISI Web of Knowledge Conference Proceedings; Index to Theses; Australasian Digital Theses.

5. Monthly searches of the NHS Economic Evaluation Database (NHS EED).

To view a list of all sources searched for ALOIS see About ALOIS on the ALOIS website.

We will run additional separate searches in many of the above sources to ensure that the most up-to-date results are retrieved. The search strategy that will be used for the retrieval of reports of trials from MEDLINE (via the OvidSP platform) can be seen in Appendix 1.

We will place no restriction on the search in respect to date of publication, risk of bias, or language of publication.

\section{Searching other resources}

We will review the reference lists of all potentially eligible papers identified and all review papers related to this topic. We will also ask the corresponding authors of each included paper to review the search results to identify any papers not initially identified from the previous searches.

We will search the conference proceedings and abstracts from the British Orthopaedic Association Annual Congress, the European Federation of National Associations of Orthopaedics and Traumatology (EFORT), the British Hip Society, and British Trauma Society meetings. We will access these through the Journal of Bone \& Joint Surgery (British Volume) Orthopaedic Proceedings. We will additionally search the INSIDE (British Library database of conference proceedings and journals).

\section{Data collection and analysis}

Effectiveness of post-operative management strategies for adults with dementia following hip fracture surgery (Protocol) 


\section{Selection of studies}

Two review authors (TS and YH) will search the results of the search strategy. They will independently review the titles and abstracts of each citation. We will order the full text version of each potentially eligible trial. This will then be assessed independently by two review authors (TS and YH) in order to re-assess its eligibility. We will include all full text papers which still satisfy the eligibility criteria of the review. Any disagreements with regards to study eligibility will be discussed between the two review authors (TS and $\mathrm{YH}$ ), and adjudicated by a third review author (CF).

\section{Data extraction and management}

We will review each study which satisfies the eligibility criteria and its data will be extracted from the original publication independently by two review authors (TS and $\mathrm{YH}$ ). They will record the data on a pre-defined eligibility database. Data extracted will include: country of origin, publication date, number of participants receiving each intervention, gender, age, and dementia diagnosis for participants, classification or type of femoral fracture, fracture fixation method, interval between fracture and surgical management, location of rehabilitation and post-operative management for each intervention, the post-operative management allocated to each group assessed, duration of intervention, follow-up period, outcome measurements used, and results from each intervention group during each follow-up period.

Disagreements between the review authors (TS and $\mathrm{YH}$ ) will be resolved through discussion. If agreement is not reached, this will be adjudicated by a third review author (CF). All agreed data will then be tabulated into a single document on Review Manager version 5.1 .

\section{Assessment of risk of bias in included studies}

The quality of the included studies and their risk of bias will be evaluated using the Cochrane Collaboration 'Risk of bias' assessment tool (Higgins 2011). For each study, we will assess: sequence generation; allocation concealment; blinding; completeness of outcome data; and selective outcome reporting. For each domain, an assessment will be made of whether there is a low risk of bias (if the study matches the criteria), a high risk (if the study does not match the criteria), or unclear risk of bias (due to underreporting).

Risk of bias will be conducted independently by two review authors (TS and YH). Any disagreement on the risk of bias scoring will be resolved through discussion. If agreement cannot be reached, this will be adjudicated by a third review author (CF).

\section{Measures of treatment effect}

We will assess whether meta-analysis is appropriate based on the heterogeneity of the study characteristics. When there is consid- erable variability between studies in respect to population, intervention, or follow-up procedure characteristics, we will perform a narrative review to summarise the treatment effect. When there is minimal or no heterogeneity between studies based on the study characteristics, we will conduct a pooled (meta-) analysis.

We will use a random-effects statistical model when $\mathrm{I}^{2}$ equates to more than $20 \%$, or the $\mathrm{Chi}^{2} \mathrm{P}$ value is greater than 0.1 . We will undertake a fixed-effect statistical model when $\mathrm{I}^{2}$ equates to less than or equal to $20 \%$ or $\mathrm{Chi}^{2}$ has a $\mathrm{P}$ value less than or equal to 0.1 . For each meta-analysis, we will calculate mean differences or standardised mean difference for continuous outcome data. We will calculate odd ratio statistics for dichotomous outcome data. We will present all meta-analysis results with $95 \%$ confidence intervals, and present forest plots.

\section{Unit of analysis issues}

The individual participant will be the unit of analysis in this review, with the exception of cluster-randomisation trial where the unit of analysis will be the specific, randomised cluster. Some grouping of follow-up periods is anticipated. Therefore, we will present the results of short-term outcomes (randomisation to six post-operative weeks), mid-term (three months to 12 months post-randomisation), and longer-term outcomes (18 months onwards). This will reduce the risk of multiplicity of results (Deeks 2011).

\section{Dealing with missing data}

We will contact corresponding authors regarding any missing data from trials included in the review. When data are unavailable after contacting the corresponding author, we will acknowledge this. We will not impute missing outcome data for any outcomes. In the event of a study only providing imputed data, we will request that the corresponding author provide data on outcomes only from the participants who were assessed rather than estimated through imputation.

\section{Assessment of heterogeneity}

We will evaluate study characteristic heterogeneity and statistical heterogeneity. We will assess study characteristic heterogeneity by examining the data extraction tables. Two review authors (TS and CF) will examine the data extraction table and assess the data for between-study variability with respect to population diagnosis, interventions (pre- and post-surgical), and outcome measurements. We will assess statistical heterogeneity for each metaanalysis through a visual assessment of the forest plot results in addition to evaluating the $\mathrm{Chi}^{2}$ test and $\mathrm{I}^{2}$ statistic. In accordance with Deeks et al (Deeks 2011), we will interpret a Chi ${ }^{2}$ test as significant with a $\mathrm{P}$ value of $0.10 . \mathrm{I}^{2}$ will be interpreted as: $0 \%$ to $40 \%$ not being important, $30 \%$ to $60 \%$ representing moderate heterogeneity, $50 \%$ to $90 \%$ representing substantial heterogeneity, and $75 \%$ to $100 \%$ representing considerable heterogeneity (Deeks 
2011). As recommended by Deeks et al (Deeks 2011), we will interpret both the $\mathrm{Chi}^{2}$ test and $\mathrm{I}^{2}$ statistic together to inform an overall assessment of statistical heterogeneity.

\section{Assessment of reporting biases}

When data are available from at least 10 studies which form a meta-analysis for a specific outcome measurement, we will generate funnel plots to assess the risk of publication bias (Sterne 2011).

\section{Data synthesis}

Two review authors (TS and CF) will evaluate study characteristic heterogeneity using the data extraction tables. When substantial in respect to the intervention, population, or method of assessment, we will present a narrative review of the results. If study characteristics heterogeneity is deemed not substantial, with homogeneity in relation to the intervention, population, or method of assessment, we will conduct meta-analyses.

\section{Subgroup analysis and investigation of heterogeneity}

If heterogeneity is identified for a priori characteristics that are included in a meta-analysis, we will undertake subgroup analyses. This may include, when appropriate, an assessment of the difference in outcomes dependent on the following.

- The severity of dementia presented, when appropriate.

Through this, we will use an assessment of dementia based on, for example, the MMSE (e.g., mild: 19 to 16; moderate: 15 to 10; severe: 9 to untestable) to compare clinical outcomes for the post-operative recovery strategies for those with greater compared to less dementia.

- Age of participant in years e.g., 60 to $69 ; 70$ to $79 ; 80$ to 89; 90 years and older.

- The type of dementia e.g., Alzheimer's disease, vascular dementia, Lewy Body, or a rarer syndrome.
- Location of intervention provision e.g., in-patient, outpatient, or home-based.

We will assess heterogeneity through examination of the data extraction tables to evaluate study characteristic heterogeneity, and using $\mathrm{I}^{2}$ and $\mathrm{Chi}^{2}$ statistics to evaluate statistical heterogeneity. Two review authors (TS and CF) will do this.

\section{Sensitivity analysis}

We will conduct sensitivity analyses if there are sufficient data to explore the influence of the following factors.

- The risk of bias: the analysis of data with the exclusion of results from studies which demonstrated a high risk of bias based on the Cochrane Collaboration's risk of bias tool (Higgins 2011).

- The analysis of data solely from published, peer-reviewed papers.

\section{'Summary of findings' Tables}

We shall use the GRADE approach to assess the quality of the body of evidence related to the primary outcome measure and the first four secondary outcome measures identified in the Types of outcome measures section.

We will construct a 'Summary of findings' (SoF) table using the GRADE software (Schunemann 2011). Using this software, the quality of the evidence can be considered along with the magnitude of the intervention's effect for each outcome of interest (Schunemann 2011). This will be used to aid interpretation of the main findings of the review.

\section{ACKNOWLEDGEMENTS}

We thank Jenny McCleery and Sue Marcus for their assistance in the preparation of this protocol, and Anna Noel-Storr for her assistance in developing the proposed search strategy.

\section{REFER E NCES}

\section{Additional references}

\section{Adams 2012}

Adams AL, Shi J, Takayanagi M, Dell RM, Funahashi TT, Jacobsen SJ. Ten-year hip fracture incidence rate trends in a large California population, 1997-2006. Osteoporosis International 2012;In Press.

Adunsky 2003

Adunsky A, Levy R, Heim M, Mizrahi E, Arad M. The unfavorable nature of preoperative delirium in elderly hip fractured patients. Archives of Gerontology and Geriatrics 2003;36:67-74.

\section{Al-Ani 2010}

Al-Ani AN, Flodin L, Söderqvist A, Ackermann P, Samnegård E, Dalén N, et al.Does rehabilitation matter in patients with femoral neck fracture and cognitive impairment? A prospective study of 246 patients. Archives of Physical Medicine and Rehabilitation 2010;91:51-7.

Allen 2012

Allen J, Koziak A, Buddingh S, Liang J, Buckingham J, Beaupre LA. Rehabilitation in patients with dementia following hip fracture: A systematic review. Physiotherapy Canada 2012;64:190-201. 


\section{American Psychiatric Association 1994}

American Psychiatric Association. Diagnostic and Statistical Manual of Mental Disorders (DSM-IV). Washington DC: American Psychiatric Association, 1994.

\section{Brandt 1991}

Brandt J. The Hopkins Verbal Learning Test: development of a new verbal memory test with six equivalent forms. The Clinical Neuropsychologist 1991;5:125-42.

\section{Bucks 1996}

Bucks RS, Ashworth DL, Wilcock GK, Siegfried K. Assessment of activities of daily living in dementia development of the Bristol Activities of Daily Living Scale. Age and Ageing 1996;25:113-20.

\section{Cameron 2000}

Cameron I, Crotty M, Currie C, Finnegan T, Gillespie L, Gillespie W, et al.Geriatric rehabilitation following fractures in older people: a systematic review. Health Technology Assessments 2000;4:1-111.

\section{Cohen-Mansfield 1986}

Cohen-Mansfield J. Agitated behaviors in the elderly. II. Preliminary results in the cognitively deteriorated. Journal of the American Geriatric Society 1986;34:722-7.

\section{Cummings 1994}

Cummings J, Mega M, Gray K, Rosenberg-Thompson S, Carusi DA, Gornbein J. The Neuropsychiatric Inventory: Comprehensive assessment of psychopathology in dementia. Neurology 1994;44:2308-14.

\section{Cummings 2002}

Cummings SR, Melton LJ. Epidemiology and outcomes of osteoporotic fractures. Lancet 2002;359:1761-7.

\section{Dawson 1996}

Dawson J, Fitzpatrick R, Carr A, Murray D. Questionnaire on the perceptions of patients about total hip replacement. Journal of Bone and Joint Surgery - British Edition 1996;78: $185-90$.

\section{Deeks 2011}

Deeks JJK, Higgins JPT, Altman DG. Chapter 9: Analysing data and undertaking meta-analysis. In: Higgins JPT, Green S editor(s). Cochrane Handbook for Systematic Reviews. Chichester, UK: Wiley-Blackwell, 2011:243-96.

\section{Drummond 2005}

Drummond M, Sculpher MJ, Torrance GW, O’Brien B, Stoddart GL. Methods for the Economic Evaluation of Health Care Programmes. Oxford: Oxford University Press, 2005.

\section{Dy 2012}

Dy CJ, Dossous PM, Ton QV, Hollenberg JP, Lorich DG, Lane JM. The medical orthopaedic trauma service: an innovative multidisciplinary team model that decreases in-hospital complications in patients with hip fractures. Journal of Orthopaedic Trauma 2012;26:379-83.

\section{Egbert 1996}

Egbert AM. Postoperative pain management in the frail elderly. Clinical Geriatric Medicine 1996;12:583-99.

\section{EuroQol Group 1990}

EuroQol Group. EuroQol-a new facility for the measurement of health-related quality of life. Health Policy 1990;16:199-208.

\section{Feeny 2002}

Feeny D, Furlong W, Torrance GW, Goldsmith CH, Zhu $\mathrm{Z}$, DePauw $\mathrm{S}$, et al.Multiattribute and single-attribute utility functions for the health utilities index mark 3 system. Medical Care 2002;40:113-28.

\section{Feldt 1998}

Feldt KS, Ryden MB, Miles S. Treatment of pain in cognitively impaired compared with cognitively intact older patients with hip-fracture. Journal of the American Geriatric Society 1998;46:1079-85.

\section{Folstein 1975}

Folstein M, Folstein S, McHugh P. "Mini-Mental State" A practical method for grading the cognitive state of patients for the clinician. Journal of Psychiatric Research 1975;12: 189-98.

\section{Gruber-Baldini 2003}

Gruber-Baldini AL, Zimmerman S, Morrison RS, Grattan LM, Hebel JR, Dolan MM, et al.Cognitive impairment in hip fracture patients: timing of detection and longitudinal follow-up. Journal of the American Geriatric Society. 2003; 51:1227-36.

\section{Handoll 2009}

Handoll HH, Cameron ID, Mak JC, Finnegan TP. Multidisciplinary rehabilitation for older people with hip fractures. Cochrane Database of Systematic Reviews 2009;4: CD007125.

\section{Hashmi 2004}

Hashmi MA, Tellisi N, Rigby AS, Wahab KH. The value of a prognostic scoring system in the rehabilitation of elderly patients with proximal femoral fractures. International Journal of Clinical Practice 2004;58:2-5.

Henderson 2007

Henderson C, Malley J, Knapp M. Maintaining good health for older people with dementia who experience fractured neck of femur. London: National Audit Office: Personal Social Services Research Unit, London School of Economics and Political Science, 2007.

Hershkovitz 2010

Hershkovitz A, Polatov I, Beloosesky Y, Brill S. Factors affecting mortality of frail hip-fractured elderly patients. Archives of Gerontology and Geriatrics 2010;51:113-6.

\section{Higgins 2011}

Higgins JPT, Altman DG. Assessing risk of bias in included studies. In: Higgins JPT, Green S editor(s). Cochrane Handbook for Systematic Reviews. Chichester, UK: WileyBlackwell, 2011:187-242.

\section{Hodkinson 1972}

Hodkinson HM. Evaluation of a mental test score for assessment of mental impairment in the elderly. Age and Ageing 1972;1:233-8. 
Hunt 2009

Hunt GR, Crealey G, Murthy BVS, Hall GM, Constantine P, O'Brien S, et al.The consequences of early discharge after hip arthroplasty for patient outcomes and healthcare costs: comparison of three centres with differing durations of stay. Clinical Rehabilitation 2009;23:1067-77.

\section{Huusko 2000}

Huusko TM, Karppi P, Avikainen V, Kautiainen H, Sulkava R. Randomised, clinically controlled trial of intensive geriatric rehabilitation in patients with hip fracture: subgroup analysis of patients with dementia. BMJ 2000; 321:1107-11.

\section{Jameson 2012}

Jameson SS, Khan SK, Baker P, James P, Gray A, Reed MR, Deehan DJ. A national analysis of complications following hemiarthroplasty for hip fracture in older patients. QJM 2012;105:55-60.

\section{Jorm 1989}

Jorm AF, Jacomb PA. The Informant Questionnaire on Cognitive Decline in the Elderly (IQCODE): Sociodemographic correlates, reliability, validity and some norms. Psychological Medicine 1989;19:1015-22.

\section{Kammerlander 2010}

Kammerlander C, Roth T, Friedman SM, Suhm N, Luger TJ, Kammerlander-Knauer U, et al.Ortho-geriatric servicea literature review comparing different models. Osteoporosis International 2010;21 Suppl 4:S637-46.

\section{Knapp 2007}

Knapp M, Privette A. Dementia UK Alzheimer's Society. London: Alzheimer's Society, 2007.

\section{Lefebvre 2011}

Lefebvre C, Manheimer E, Glanville J. Chapter 6: Searching for studies. In: Higgins JPT, Green S editor(s). Cochrane Handbook for Systematic Reviews. Chichester, UK: WileyBlackwell, 2011:96-150.

\section{Lenze 2007}

Lenze EJ, Skidmore ER, Dew MA, Butters MA, Rogers JC, Begley A, et al.Does depression, apathy or cognitive impairment reduce the benefit of inpatient rehabilitation facilities for elderly hip fracture patients?. General Hospital Psychiatry 2007;29:141-6.

Lieberman 2006

Lieberman D, Friger M, Lieberman D. Inpatient rehabilitation outcome after hip fracture surgery in elderly patients: a prospective cohort study of 956 patients. Archies of Physical Medicine and Rehabilitation 2006;87:167-71.

\section{Magaziner 1990}

Magaziner J, Simonsick EM, Kashner TM, Hebel JR, Kenzora JE. Predictors of functional recovery one year following hospital discharge for hip fracture: a prospective study. Journal of Gerontology 1990;45:M101-7.

\section{Magaziner 2002}

Magaziner J, Hawkes W, Hebel JR, Zimmerman SI, Fox $\mathrm{KM}$, Dolan $\mathrm{M}$, et al.Recovery from hip fracture in eight areas of function. Journal of Gerontology. Series A. Biological Sciences and Medical Sciences 2002;55:M498-507.

\section{Mahoney 1965}

Mahoney F, Barthel D. Functional evaluation: the Barthel Index. Maryland Medical Journal 1965;14:61-5.

\section{Mathuranath 2005}

Mathuranath PS, Nestor PJ, Berrios GE, Rakowicz W, Hodges JR. A brief cognitive test battery to differentiate Alzheimer's disease and frontotemporal dementia. Neurology 2000;55:1613-20.

\section{McGilton 2012}

McGilton KS, Davis A, Mahomed N, Flannery J, Jaglal $S$, Cott $\mathrm{C}$, et al.An inpatient rehabilitation model of care targeting patients with cognitive impairment. $B M C$ Geriatrics 2012;12:21.

\section{Morrison 1998}

Morrison RS, Ahronheim JC, Morrison GR, Darling E, Baskin SA, Morris J, et al.Pain and discomfort associated with common hospital procedures and experiences. Journal of Pain and Symptom Management 1998;15:91-101.

\section{Morrison 2000}

Morrison RS, Siu AL. A comparison of pain and its treatment in advanced dementia and cognitively intact patients with hip fracture. Journal of Pain and Symptom Management 2000;19:240-8.

\section{Nasreddine 2005}

Nasreddine ZS, Phillips NA, Bédirian V, Charbonneau $S$, Whitehead V, Collin I, et al.The Montreal Cognitive Assessment (MoCA): A brief screening tool for mild cognitive impairment. Journal of the American Geriatrics Society 2005;53:695-9.

\section{NICE 2011}

NICE. Hip fracture: The management of hip fracture in adults. Available at: http://publications.nice.org.uk/hipfracture-cg124 2011.

\section{Nouri 1987}

Nouri FM, Lincoln NB. An extended activities of daily living scale for stroke patients. Clinical Rehabilitation 1987; 1:301-5.

\section{Parker 2010}

Parker MJ, Handoll HHG. Gamma and other cephalocondylic intramedullary nails versus extramedullary implants for extracapsular hip fractures in adults. Cochrane Database of Systematic Reviews 2010;9:CD000093.

\section{Rosen 1984}

Rosen WG, Mohs RC, Davis KL. A new rating scale for Alzheimer's disease. American Journal of Psychiatry 1984; 141:1356-64.

\section{Schunemann 2011}

Schunemann HJ, Oxman AD, Higgins JPT, Glasziou P, Guyatt GH. Presenting results and 'Summary of findings' tables. In: Higgins JPT, Green S editor(s). Cochrane Handbook of Systematic Reviews. Chichester, UK: WileyBlackwell, 2011:335-57.

\section{Shemilt 2008}

Shemilt I, Mugford M, Byford S, Drummond M, Eisenstein E, Knapp M, et al.Chapter 15: Incorporating 
economics evidence. In: Higgins JPT, Green S editor (s). Cochrane Handbook for Systematic Reviews of Interventions. Version 5.0.1 [updated September 2008]. The Cochrane Collaboration: Available from www.cochranehandbook.org, 2008.

\section{Smith 2005}

Smith SC, Lamping DL, Banerjee S, Harwood R, Foley B, Smith P, et al.Measurement of health-related quality of life for people with dementia: development of a new instrument (DEMQOL) and an evaluation of current methodology.

Health Technology Assessment 2005;9:1-93.

\section{Steiner 1997}

Steiner JF, Kramer AM, Eilertsen TB, Kowalsky JC. Development and validation of a clinical prediction rule for prolonged nursing home residence after hip fracture. Journal of the American Geriatric Society 1997;45:1510-4.

\section{Stenvall 2012}

Stenvall M, Berggren M, LundströmM, Gustafson Y, Olofsson B. A multidisciplinary intervention program improved the outcome after hip fracture for people with dementia--subgroup analyses of a randomized controlled trial. Archives of Gerontology and Geriatrics 2012;54:e284-9.

\section{Sterne 2011}

Sterne J, Egger M, Moher D. Chapter 10: Addressing reporting bias. In: Higgins JPT, Green S editor(s). Cochrane Handbook for Systematic Reviews. Chichester, UK: WileyBlackwell, 2011.

\section{Söderqvist 2007}

Söderqvist A, Ponzer S, Tidermark J. Cognitive function and pressure ulcers in hip fracture patients. Scandinavian Journal of Caring Sciences 2007;21:79-83.

Trigg 2007

Trigg R, Skevington, SM, Jones RW. How can we best assess the quality of life of people with dementia? The
Bath Assessment of Subjective Quality of Life in Dementia (BASQID). The Gerontologist 2007;47:789-97.

\section{Uzoigwe 2012}

Uzoigwe CE, Burnand HG, Cheesman CL, Aghedo DO, Faizi M, Middleton RG. Early and ultra-early surgery in hip fracture patients improves survival. Injury 2013;44(6): 726-9.

\section{Vidal 2012}

Vidal EI, Moreira-Filho DC, Pinheiro RS, Souza RC, Almeida LM, Camargo KR Jr, et al.Delay from fracture to hospital admission: a new risk factor for hip fracture mortality?. Osteoporosis International 2013; In Press.

Warden 2003

Warden V, Hurley AC, Volicer V. Development and psychometric evaluation of the Pain Assessment in Advanced Dementia (PAINAD) scale. Journal of American Medical Directors Association 2003;4:9-15.

\section{Ware 1992}

Ware JE Jr, Sherbourne CD. The MOS 36-item short-form health survey (SF-36). I. Conceptual framework and item selection. Medical Care 1992;30:473-83.

\section{Ware 1996}

Ware JE, Kosinski M, Keller SD. A 12-item short-form health survey. Medical Care 1996;34:220-3.

\section{World Health Organization 2007}

World Health Organization. International Statistical Classification of Diseases and Related Health Problems. 10th Revision. http://apps.who.int/classifications/apps/icd/ icd10online/ 20072007.

\section{Zhao 2012}

Zhao Y, Shen L, Ji H-F. Alzheimer's disease and risk of hip fracture. The Scientific World Journal 2012;872173:1-5.

* Indicates the major publication for the study

\section{A P P E N D I C ES}

\section{Appendix I. MEDLINE search strategy}

1. exp Dementia/

2. Delirium/

3. Wernicke Encephalopathy/

4. Delirium, Dementia, Amnestic, Cognitive Disorders/

5. dement*.mp.

6. alzheimer*.mp.

7. (lewy* adj2 bod*).mp.

8. deliri*.mp.

9. (chronic adj2 cerebrovascular).mp. 
10. ("organic brain disease" or "organic brain syndrome").mp.

11. ("normal pressure hydrocephalus" and "shunt*").mp.

12. "benign senescent forgetfulness".mp.

13. (cerebr* adj2 deteriorat*).mp.

14. (cerebral* adj2 insufficient*).mp.

15. (pick* adj2 disease).mp.

16. (creutzfeldt or jcd or cjd).mp.

17. huntington*.mp.

18. binswanger*.mp.

19. korsako*.mp.

20. or/1-19

21. exp Femur/

22. exp Fractures, Bone/

23. exp Fracture Fixation/

24. exp Fracture Healing/

25. or/ $22-24$

26. 21 and 25

27. (hip or hips or pertrochant* or intertrochant* or trochanteric or subtrochanteric or extracapsular*).ti,ab.

28. ((femur* or femoral*) adj3 (neck or proximal)).ti,ab.

29. 27 or 28

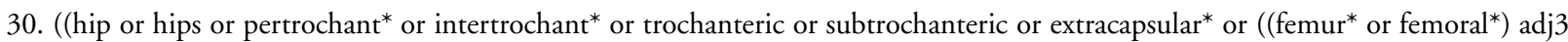
(neck or proximal))) adj4 fracture).ti,ab.

31. randomized controlled trial.pt.

32. controlled clinical trial.pt.

33. randomi?ed.ab.

34. randomly.ab.

35. placebo.ab.

36. drug therapy.fs.

37. trial.ab.

38. groups.ab.

39. ("double-blind"” or "single-blind"”).ti,ab.

40. (RCT or CCT).ti,ab.

41. or/31-40

42. (animals not (humans and animals)).sh.

43. 41 not 42

44. 29 or 30

45. 20 and 43 and 44

\section{CONTRIBUTIONS OFAUTHORS}

TS: drafted the protocol; develop and run the search strategy; reviewed and approved final protocol; act as guarantor.

YH: drafted the protocol; reviewed and approved final protocol.

$\mathrm{CH}$ : drafted the protocol; reviewed and approved final protocol.

JC: drafted the protocol; reviewed and approved final protocol.

OS: drafted the protocol; reviewed and approved final protocol.

CF: drafted the protocol; reviewed and approved final protocol.

Effectiveness of post-operative management strategies for adults with dementia following hip fracture surgery (Protocol) 


\section{DECLARATIONSOF INTEREST}

No conflicts of interests have been declared by any of the authors in relation to this protocol.

\section{SOURCES OF SUPPORT}

\section{Internal sources}

- No sources of support supplied

\section{External sources}

- National Institute for Health Research, UK.

This review will form part of a NIHR Programme Grant (Reference Number: DTC-RP-PG-0311-10004; Chief Investigator: Fox) 